\title{
The Stability of Highly Pathogenic Avian Influenza Epidemic Model with Saturated Contact Rate
}

\author{
Shuqin Che ${ }^{1 *}$, Yakui Xue1, Likang $\mathrm{Ma}^{2}$ \\ ${ }^{1}$ Department of Mathematics, North University of China, Taiyuan, China \\ 2 Jinxi Industries Group Co., Ltd., Taiyuan, China \\ Email: ${ }^{*}$ 2641511342@qq.com
}

Received 29 September 2014; revised 28 October 2014; accepted 18 November 2014

Copyright (C) 2014 by authors and Scientific Research Publishing Inc.

This work is licensed under the Creative Commons Attribution International License (CC BY).

http://creativecommons.org/licenses/by/4.0/

(c) (i) Open Access

\begin{abstract}
In this paper we present a highly pathogenic Avian influenza epidemic model with saturated contact rate. According to study of the dynamics, we calculated the basic reproduction number of the model. Through the analysis of this model, we have the following conclusion: if $R_{0} \leq 1$, there is only one disease-free equilibrium which is globally stable, the disease will die; if $R_{0}>1$, there is only one endemic equilibrium which is globally stable, disease will be popular.
\end{abstract}

\section{Keywords}

Avian Influenza, The Basic Reproduction Number, Stability

\section{Introduction}

Avian influenza virus belongs to the influenza A virus. According to the difference of the pathogenic Avian influenza virus in chicken and turkey, we divided it into three levels: high, medium, low/non pathogenic. Because of the bird flu virus hemagglutinin structure characteristics, general infected birds, when the virus genetic reassortment during replication, causing structural changes. The Avian influenza virus which acquires the ability to infect people, can make adult infect the Avian influenza disease. The highly pathogenic Avian influenza has a high death rate, which is about 100 percent for birds and more than 70 percent for humans [1].

Up to now, we found that Avian influenza virus subtypes which can directly infect human are: $H 5 N 1, H 7 N 1$, $H 7 N 2$, $H 7 N 3$, H7N7, H9N2, H7N9, subtype. Among them, the new subtype Avian influenza virus $H 7 N 9$ subtype, which was first discovered in 2013 March, and the high pathogenic Avian influenza H5N1 subtype, are

*Corresponding author. 
particularly noteworthy. The Avian influenza virus not only caused human casualties, but also hit the poultry industry.

Avian influenza virus (H5N1 subtype Avian influenza virus) had 12 human infections in Hongkong, including 6 deaths. According to the WHO's statistics, 393 people around the world in more than 15 countries and regions were infected, including 248 deaths; the mortality rate was 0.63 . Since 2003, 31 people have been infected with Avian influenza in China, including 21 deaths. Although people infection probability is not high, the mortality rate is very high. By March 2013, the world has reported a total of human infection of highly pathogenic H5N1 Avian influenza in 622 cases, including 371 deaths. The distribution of cases in 15 countries, including China, is found in 45 cases, 30 cases of death. Most of human infections with $H 5 N 1$ Avian influenza are young people and children. In March 2013, human infection with H7N9 Avian influenza was first found in China. By May 1, 2013, Shanghai, Anhui, Jiangsu, Zhejiang, Beijing, Henan, Shandong, Jiangxi, Hunan, Fujian and other 10 provinces (city) have reported 127 confirmed cases, including 26 death cases. The cases in the majority are with the elderly, men more than women [2].

The present study found that people are infected with bird flu infection for the virus's birds, but the transmission is still to be defined. Research suggests that the main way of human infection with the H5N1 Avian influenza is in close contact with poultry, high-risk behavior, including the slaughter, feathers and processing of infected poultry. In a few cases, when children frequently play in the backyard poultry regional, the exposure poultry manure was also thought to be a source of infection. At present, most of the researched evidences indicate the presence of bird-human transmission; there may be an environment-human (birds excrement pollution) transmission, and a few non sustained human-human transmission of H5N1. Now H7N9 Avian influenza patients are infected through direct contact with poultry or the items, environment which are contaminated by feces. Human infection with H7N9 Avian influenza is still sporadic, although there have been cases of aggregation of individual families; but it is not currently found that the virus has sustained human-human transmission capacity.

At present, some authors have researched some Avian influenza model [3] [4], they had constructed a mathematical model which interprets the spread of Avian influenza from the bird world to the human world. Literature [5] has introduced a piecewise treatment function. When the number of the infective had not exceeded the maximum treatment capacity, the treatment rate was proportional to the number of the infective. When the number of the infective had exceeded the maximum treatment capacity, it took maximum saturation treatment value. Literature [6]-[8] have studied of SIR model with saturated treatment rate. In the literature [8], the saturated treatment rate is $\frac{r I}{1+\alpha I}$, where $r$ is the cure rate and $\alpha$ is the parameters of infection which is due to delayed treatment. The conclusion had indicated that in the prevention and treatment of Avian flu drugs under the condition of limited, culling of infected poultry was the most effective way to control the spread of Avian flu in humans. Literature [9] [10] have proposed the model of highly pathogenic Avian influenza.

In this paper, the authors propose a Avian influenza model with saturated contact rate [11],

$$
\left\{\begin{array}{l}
X^{\prime}=c-\frac{\omega X Y}{1+\delta Y}-d X, \\
Y^{\prime}=\frac{\omega X Y}{1+\delta Y}-(d+m) Y, \\
S^{\prime}=b-\frac{\beta S Y}{1+\delta Y}-\alpha S, \\
I^{\prime}=\frac{\beta S Y}{1+\delta Y}-(\varepsilon+\alpha+\gamma) I, \\
R^{\prime}=\gamma I-\alpha R .
\end{array}\right.
$$

In system (1.1), the human is divided into three compartments: Susceptible $(S)$, infected $(I)$, recovery $(R)$. The birds are divided into susceptible poultry $(X)$ and infected poultry $(Y)$. The parameters $c$ and $b$ are respectively the natural birth rate of Avian and human. $d$ and $\alpha$ are respectively the natural mortality of poultry and human. $m$ and $\varepsilon$ are respectively the poultry and human mortality due to illness. $\omega$ stands for infectious rate of susceptible poultry to infected poultry. $\beta$ stands for infected poultry of the infection rate of susceptible individuals. $\gamma$ is the recovery rate that infects individuals through treatment. When $Y$ is small, the contact ratio, infected poultry and susceptible poultry, is approximatively proportional to the $Y$; With the increase of $Y$, the con- 
tact rate gradually reaches saturation. When $Y$ is very large, it is close to a constant $\frac{\omega}{\delta}$. The same way to explain $\frac{\beta}{1+\delta Y}$, that is to say, $\delta$ is a parameter, which is effects of infectious diseases, when the contact rate of the disease is saturated. The transmission process of the disease is shown in Figure 1.

\section{The Existence of the Equilibrium Point}

The first four equations of system (1.1) do not contain $R$, by the method of van den Driessche and Watmough Diekmann [12], we only need to study the following system:

$$
\left\{\begin{array}{l}
X^{\prime}=c-\frac{\omega X Y}{1+\delta Y}-d X, \\
Y^{\prime}=\frac{\omega X Y}{1+\delta Y}-(d+m) Y, \\
S^{\prime}=b-\frac{\beta S Y}{1+\delta Y}-\alpha S, \\
I^{\prime}=\frac{\beta S Y}{1+\delta Y}-(\varepsilon+\alpha+\gamma) I .
\end{array}\right.
$$

It's easy to get a disease-free equilibrium of the system (2.1) $E_{0}\left(X^{0}, Y^{0}, S^{0}, I^{0}\right)$ is $\left(\frac{c}{d}, 0, \frac{b}{\alpha}, 0\right)$, by the method of van den Driessche and Watmough, we can get the basic reproductive number of the system (2.1)

$$
R_{0}=\frac{c \omega}{d(d+m)}
$$

By the positive of the endemic equilibrium point, we can get that if $R_{0}>1$, there is a unique endemic equilibrium $E_{+}\left(X^{*}, Y^{*}, S^{*}, I^{*}\right)$, which satisfied:

$$
\begin{gathered}
X^{*}=\frac{d+m-c \delta}{d \delta+\omega}, \quad Y^{*}=\frac{c \omega-d(d+m)}{(d+m)(d \delta+\omega)}, \quad S^{*}=\frac{b\left(1+\delta Y^{*}\right)}{\beta Y^{*}+\alpha\left(1+\delta Y^{*}\right)}, \\
I^{*}=\frac{b \beta Y^{*}}{(\varepsilon+\alpha+\gamma)\left(\beta Y^{*}+\alpha\left(1+\delta Y^{*}\right)\right)} .
\end{gathered}
$$

So, we can get the following theorem:

Theorem 2.1 If $R_{0} \leq 1$, the system (2.1) only exists the disease-free equilibrium $E_{0}\left(\frac{c}{d}, 0, \frac{b}{\alpha}, 0\right)$; when $R_{0}>1$, there exists only one endemic equilibrium

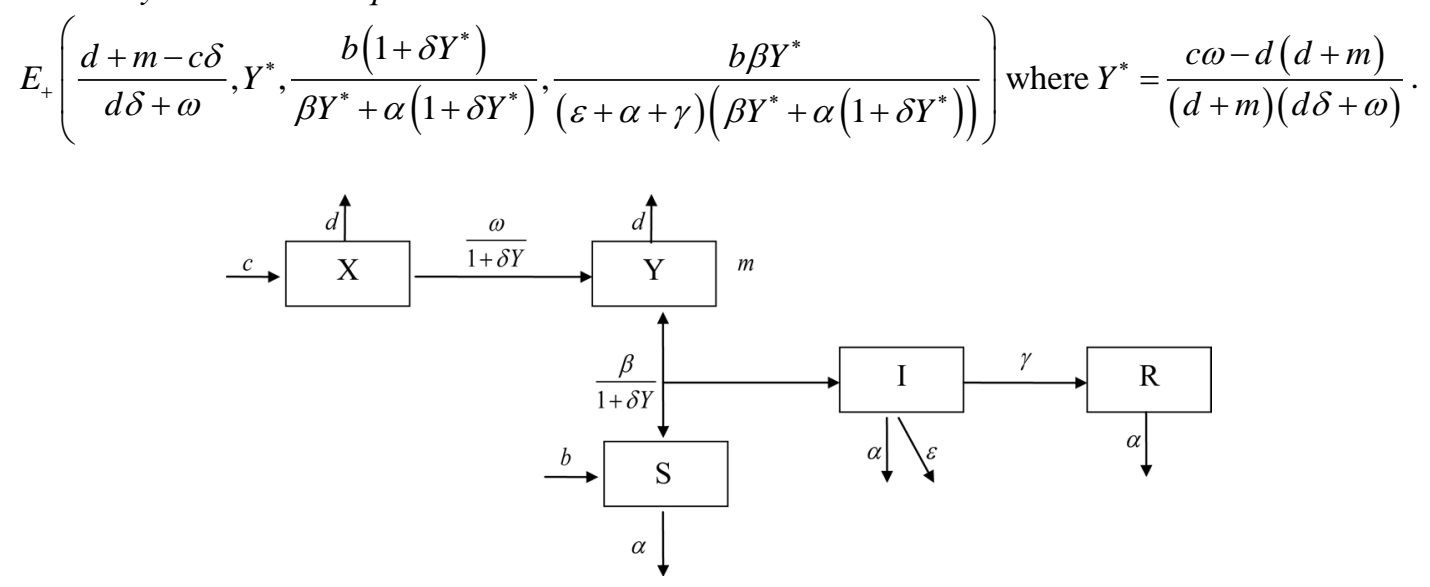

Figure 1. Dissemination flow diagram. 


\section{The Stability of the Disease-Free Equilibrium}

The Jacobian matrix of system (2.1) is

$$
J=\left(\begin{array}{cccc}
-d & -\frac{\omega X(1+\delta Y)-\delta \omega X Y}{(1+\delta Y)^{2}} & 0 & 0 \\
\frac{\omega Y}{1+\delta Y} & \frac{\omega X(1+\delta Y)-\delta \omega X Y}{(1+\delta Y)^{2}}-(d+m) & 0 & 0 \\
0 & -\frac{\beta S(1+\delta Y)-\delta \beta S Y}{(1+\delta Y)^{2}} & -\frac{\beta Y}{1+\delta Y}-\alpha & 0 \\
0 & \frac{\beta S(1+\delta Y)-\delta \beta S Y}{(1+\delta Y)^{2}} & \frac{\beta Y}{1+\delta Y} & -(\varepsilon+\alpha+\gamma)
\end{array}\right)
$$

which in the disease-free equilibrium $E_{0}$, is

$$
J_{E_{0}}=\left(\begin{array}{cccc}
-d & -\frac{c \omega}{d} & 0 & 0 \\
0 & \frac{c \omega}{d}-(d+m) & 0 & 0 \\
0 & -\frac{b \beta}{\alpha} & -\alpha & 0 \\
0 & \frac{b \beta}{\alpha} & 0 & -(\varepsilon+\alpha+\gamma)
\end{array}\right)
$$

The characteristic equation of the Jacobian matrix $J_{E_{0}}$ is

$$
(\lambda+d)(\lambda-h)(\lambda+\alpha)(\lambda+\varepsilon+\alpha+\gamma)=0 .
$$

Here, $h=\frac{c \omega}{d}-(d+m)=(d+m)\left(R_{0}-1\right), \lambda$ denotes the indeterminate of the polynomial. If and only if $R_{0} \leq$ 1 , all roots of this characteristic equation have negative real parts. It implies that $E_{0}$ is locally asymptotically stable. Then we can get the following theorem:

Theorem 3.1 If $R_{0} \leq 1$, the disease-free equilibrium $E_{0}$ is locally asymptotically stable; if $R_{0}>1$, the diseasefree equilibrium $E_{0}$ is unstable.

Next, we discuss the global stability of $E_{0}$, considering the Liapunov function [13] $W_{1}=X-X^{0}-X^{0} \ln X+Y$, then

$$
\begin{aligned}
W_{1}^{\prime} & =X^{\prime}-X^{0} \frac{1}{X} X^{\prime}+Y^{\prime}=X^{\prime}\left(1-\frac{X^{0}}{X}\right)+Y^{\prime} \\
& =\left(1-\frac{X^{0}}{X}\right)\left(-d\left(X-X^{0}\right)-\frac{\omega X Y}{1+\delta Y}\right)+\frac{\omega X Y}{1+\delta Y}-(d+m) Y \\
& =-\frac{d\left(X-X^{0}\right)^{2}}{X}+\frac{\omega X^{0} Y}{1+\delta Y}-(d+m) Y \\
& =-\frac{d\left(X-X^{0}\right)^{2}}{X}+(d+m) Y\left(\frac{\omega X^{0}}{(1+\delta Y)(d+m)}-1\right) \\
& \leq-\frac{d\left(X-X^{0}\right)^{2}}{X}+(d+m) Y\left(\frac{\omega X^{0}}{d+m}-1\right) \\
& =-\frac{d\left(X-X^{0}\right)^{2}}{X}+(d+m) Y\left(R_{0}-1\right)
\end{aligned}
$$


when $R_{0} \leq 1$, we can get $W_{1}^{\prime} \leq 0$, and $W_{1}^{\prime}=0$ has no other closed trajectory in addition to $E_{0}$. Then we can get the following theorem:

Theorem 3.2 If $R_{0} \leq 1$, the disease-free equilibrium $E_{0}$ is globally asymptotically stable.

\section{The Stability of the Endemic Equilibrium}

The Jacobian matrix of system (2.1), which in the endemic equilibrium $E_{+}$, is

$$
J_{E_{+}}=\left(\begin{array}{ll}
A & 0 \\
B & C
\end{array}\right)
$$

where

$$
\begin{gathered}
A=\left(\begin{array}{cc}
-d & -\frac{\omega X^{*}\left(1+\delta Y^{*}\right)-\delta \omega X^{*} Y^{*}}{\left(1+\delta Y^{*}\right)^{2}} \\
\frac{\omega Y^{*}}{1+\delta Y^{*}} & \frac{\omega X^{*}\left(1+\delta Y^{*}\right)-\delta \omega X^{*} Y^{*}}{\left(1+\delta Y^{*}\right)^{2}}-(d+m)
\end{array}\right), \\
B=\left(\begin{array}{cc}
0 & -\frac{\beta S^{*}\left(1+\delta Y^{*}\right)-\delta \beta S^{*} Y^{*}}{\left(1+\delta Y^{*}\right)^{2}} \\
0 & \frac{\beta S^{*}\left(1+\delta Y^{*}\right)-\delta \beta S^{*} Y^{*}}{\left(1+\delta Y^{*}\right)^{2}}
\end{array}\right), \\
C=\left(\begin{array}{cc}
-\frac{\beta Y^{*}}{1+\delta Y^{*}}-\alpha & 0 \\
\frac{\beta Y^{*}}{1+\delta Y^{*}} & -(\varepsilon+\alpha+\gamma)
\end{array}\right) .
\end{gathered}
$$

Thus, $J_{E_{+}}$evaluated is stable if and only if so are $A$ and $C$. Then the characteristic equation of the matrix $A$ is

$$
\lambda^{2}+h_{1} \lambda+h_{2}=0
$$

where,

$$
h_{1}=(d+m) \frac{\delta Y^{*}}{1+\delta Y^{*}}, \quad h_{2}=(d+m) \frac{\delta Y^{*}}{1+\delta Y^{*}}\left[d+\frac{d+m}{\delta}\right] .
$$

Obviously, when $R_{0}>1, h_{1}>0, h_{2}>0$. By the Harwize criterion, it implies that the characteristic roots of matrix $A$ have negative real parts. then, we analyze the characteristics equation of the matrix $C$

$$
\left[\lambda+\alpha+\frac{\beta Y^{*}}{1+\delta Y^{*}}\right][\lambda+(\varepsilon+\alpha+\gamma)]=0 .
$$

Easily, if and only if $R_{0}>1$, all roots of this characteristic equation have negative real parts. So, we can get the following theorem:

Theorem 4.1 If $R_{0}>1$, the endemic equilibrium $E_{+}$is locally asymptotically stable.

Next, we discuss the global stability of $E_{+}$, considering the Liapunov function $W_{2}=X^{*}\left(\frac{X}{X^{*}}-1-\ln \frac{X}{X^{*}}\right)+Y^{*}\left(\frac{Y}{Y^{*}}-1-\ln \frac{Y}{Y^{*}}\right)$, then derivated $W_{2}$ about system (2.1),

$$
W_{2}^{\prime}=\left(1-\frac{X^{*}}{X}\right) X^{\prime}+\left(1-\frac{Y^{*}}{Y}\right) Y^{\prime}=c\left(2-\frac{X^{*}}{X}-\frac{X}{X^{*}}\right) .
$$


By the relationship of arithmetic mean and geometric mean, we can know $2-\frac{X^{*}}{X}-\frac{X}{X^{*}} \leq 0$. That is to say, $W_{2}^{\prime} \leq 0$. If and only if $(X, Y)=\left(X^{*}, Y^{*}\right), W_{2}^{\prime}=0$. Thus, by LaSalle Invariance Principle [14], we can get the following theorem:

Theorem 4.2 If $R_{0}>1$, the endemic equilibrium $E_{+}$is globally asymptotically stable.

\section{The Numerical Simulations}

In this section, the model has been analyzed numerically by using MAT-LAB 7.5.0, to confirm the results we have obtained. For this purpose we have used the following set of parameter values:

When $c=2 ; \beta=0.01 ; d=0.03 ; \omega=0.014 ; m=0.97 ; b=1 ; \alpha=0.069 ; \varepsilon=0.63 ; \gamma=0.301 ; \delta=0.05$, then $R_{0}=$ 0.9333 . These parameters values satisfy the condition $R_{0}<1$. Thus, the two images of Figure 2 show that if $R_{0}$ $<1$, the disease-free equilibrium $E_{0}$ is global asymptotically stable.

When $c=2 ; \beta=0.01 ; d=0.03 ; \omega=0.02 ; m=0.97 ; b=1 ; \alpha=0.069 ; \varepsilon=0.63 ; \gamma=0.301 ; \delta=0.05$ then $R_{0}=$ 1.3333; These parameters values satisfy the condition $R_{0}>1$. Thus, the two image of Figure 3 shows that if $R_{0}>$ 1 , the unique endemic equilibrium $E^{*}$ is global asymptotically stable.

\section{Conclusion}

In this paper we have discussed the stability of highly pathogenic Avian influenza epidemic model with saturated contact rate. We get the reproduction number $R_{0}$; it is the threshold which is endemic or not. If $R_{0} \leq 1$,

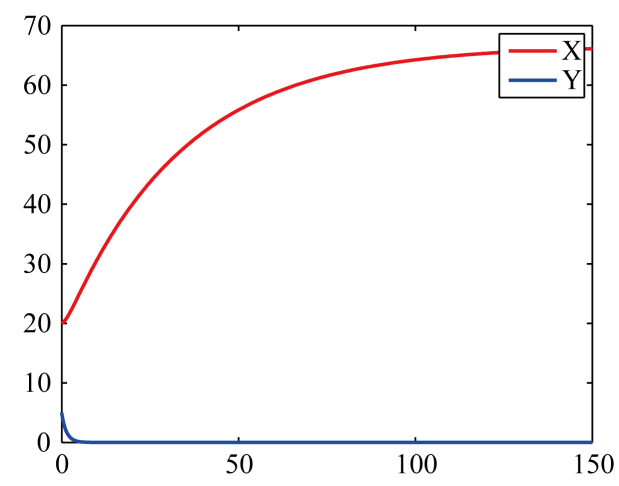

(a)

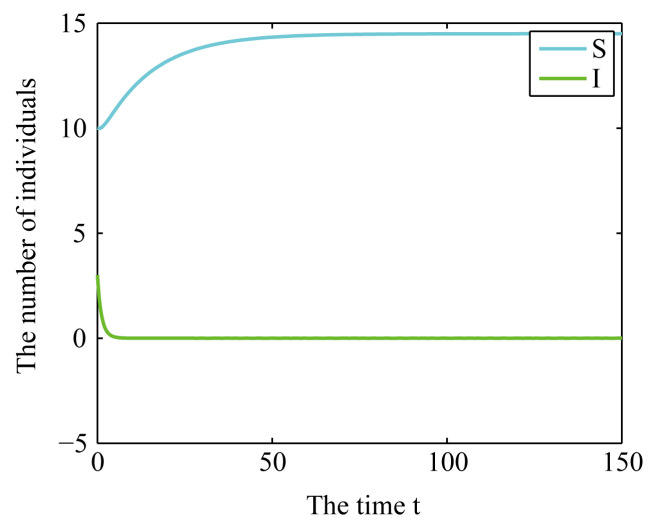

(b)

Figure 2. $c=2 ; \beta=0.01 ; d=0: 03 ; \omega=0.014 ; m=0.97 ; b=1 ; \alpha=0.069 ; \varepsilon=0.63 ; \gamma=0.301 ; \delta=0.05$; $R_{0}=0.9333$.

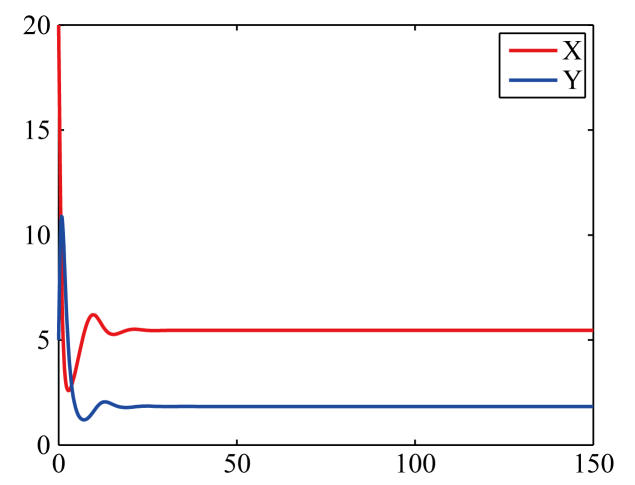

(a)

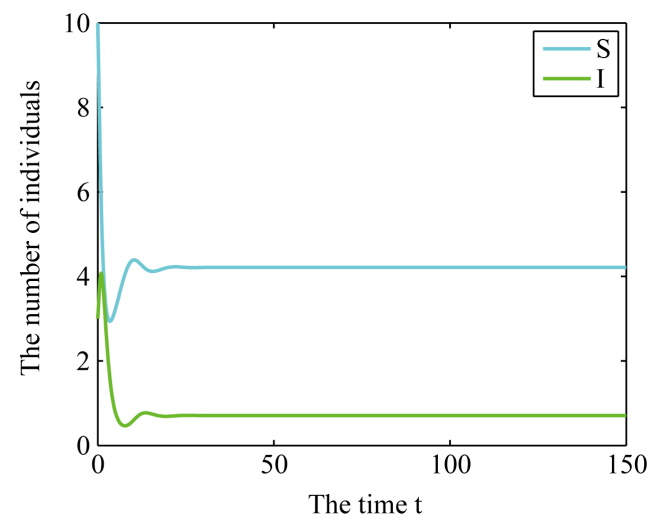

(b)

Figure 3. $c=2 ; \beta=0.01 ; d=0.03 ; \omega=0.02 ; m=0.97 ; b=1 ; \alpha=0.069 ; \varepsilon=0.63 ; \gamma=0.301 ; \delta=0.05$; $R_{0}=1.3333$. 
the disease-free equilibrium is global asymptotic stability which implies that the disease will be extinct. If $R_{0}>1$, the endemic equilibrium $E_{+}$is globally asymptotically stable, that implies that the disease will sustain and lead to epidemic disease eventually. In order to prevent epidemics, through the analysis of the model, we can get: reducing the activities of the poultry market; culling or burying the infected poultry. That the infected poultry feces get disinfected or burned can prevent the pollution of the surrounding water. The government must strictly manage cooked poultry meat, and meat sources. Traditional farming poultry from backyard changes the concentration of scale breeding, slaughter processing and scientific transport, raising poultry breeding, circulation biological safety level, thereby reduces the population of live poultry or poultry exposure opportunity. Early discovery, early diagnosis of Avian influenza are the implementation of case isolation and diagnosis and treatment timely, effectively and reasonably.

\section{References}

[1] Iwami, S., Takeuchi, Y. and Liu, X.N. (2007) Avian-Human Influenza Epidemic Model. Mathematical Biosciences, 207, 1-25. http://dx.doi.org/10.1016/j.mbs.2006.08.001

[2] Feng, Z.J. (2013) Human Infect with Avian Influenza H7N9 Treatment Program. Encyclopedia of Medical Network, 4. http://www.baikemy.com/jiankangkepu/10339395895809

[3] Iwami, S., Takeuchi, Y., Korobeinikov, A. and Liu, X. (2008) Prevention of Avian Influenza Epidemic: What Policy Should We Choose. Journal of Theoretical Biology, 252, 732-741. http://dx.doi.org/10.1016/j.mbs.2006.08.001

[4] Rao, D.M., Chernyakhovsky, A. and Rao, V. (2009) Modeling and Analysis of Global Epidemiology of Avian Influenza. Environmental Modeling \& Software, 24, 124-134. http://dx.doi.org/10.1016/j.envsoft.2008.06.011

[5] Wang, W. (2006) Backward Bifurcation of an Epidemic Treatment. Mathermatical Biosciences, 201, 58-71. http://dx.doi.org/10.1016/j.mbs.2005.12.022

[6] Zhang, X. and Liu, X. (2008) Backford Bifurcation of an Epidemic Model with Saturated Treatment Function. Journal of Mathematical Analysis and Applications, 348, 433-443. http://dx.doi.org/10.1016/j.jmaa.2008.07.042

[7] Capasso, V. and Serio, G. (1978) A Generalization of the Kermack-Mckendrick Deterministic Epidemic Model. Mathematical Biosciences, 42, 43-61. http://dx.doi.org/10.1016/0025-5564(78)90006-8

[8] Guo, S.M., Guo, L.N. and Li, X.Z. (2010) Analysis of an Avian Influenza Epidemic Model with Saturation Treatment. Mathematics in Practice and Theory, 40, 134-137.

[9] Li, Y.-L. (2009) Study on SI Transmission Model of Highly Pathogenic Avian Influenza. Journal of Anhui Agricultural Sciences, 37, 13603-13605.

[10] Liang, R.H. (2007) The Construction and Application of SEID Model for the Disseminate Mechanism of Highly Pathogenic Avian Influenza (HPAI). Journal of Xinyang Normal University: Natural Science Edition, 20, 262-265.

[11] Lasalle, J.P. (1986) The Stability and Control of Discrete Process. Springer-Verlag, New York. http://dx.doi.org/10.1007/978-1-4612-1076-4

[12] van den Driessche, P. and Watmough, J. (2007) Reproduction Numbers and Sub-Threshold Endemic Equilibria for Compartmental Models of Disease Transmission. Mathematical Biosciences, 196, 1679-1684.

[13] Ma, Z.-E. and Zhou, Y.-C. (2001) The Theory of Ordinary Differential Equation Qualitative and Stability. Science Press, Beijing.

[14] Lasalle, J.P. (1976) The Stability of Dynamical Systems. Regional Conference Series in Applied Mathematics Philadelphia, SIAM, 21, 418-420. 
Scientific Research Publishing (SCIRP) is one of the largest Open Access journal publishers. It is currently publishing more than 200 open access, online, peer-reviewed journals covering a wide range of academic disciplines. SCIRP serves the worldwide academic communities and contributes to the progress and application of science with its publication.

Other selected journals from SCIRP are listed as below. Submit your manuscript to us via either submit@scirp.org or Online Submission Portal.
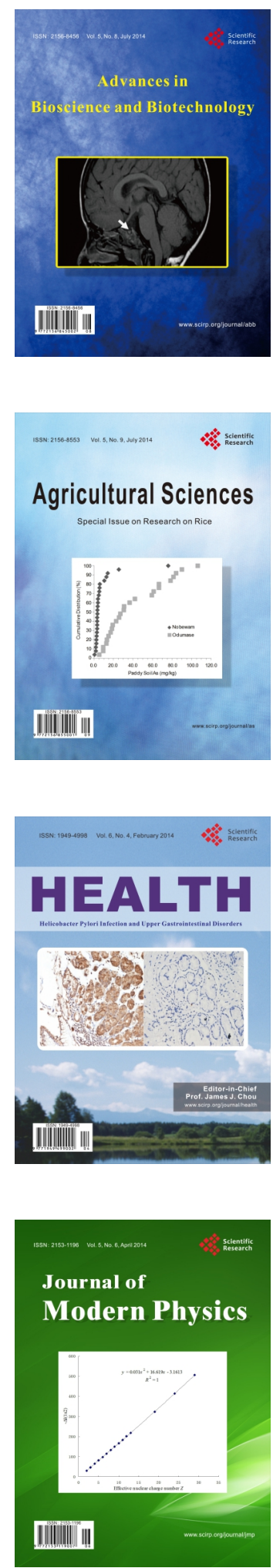
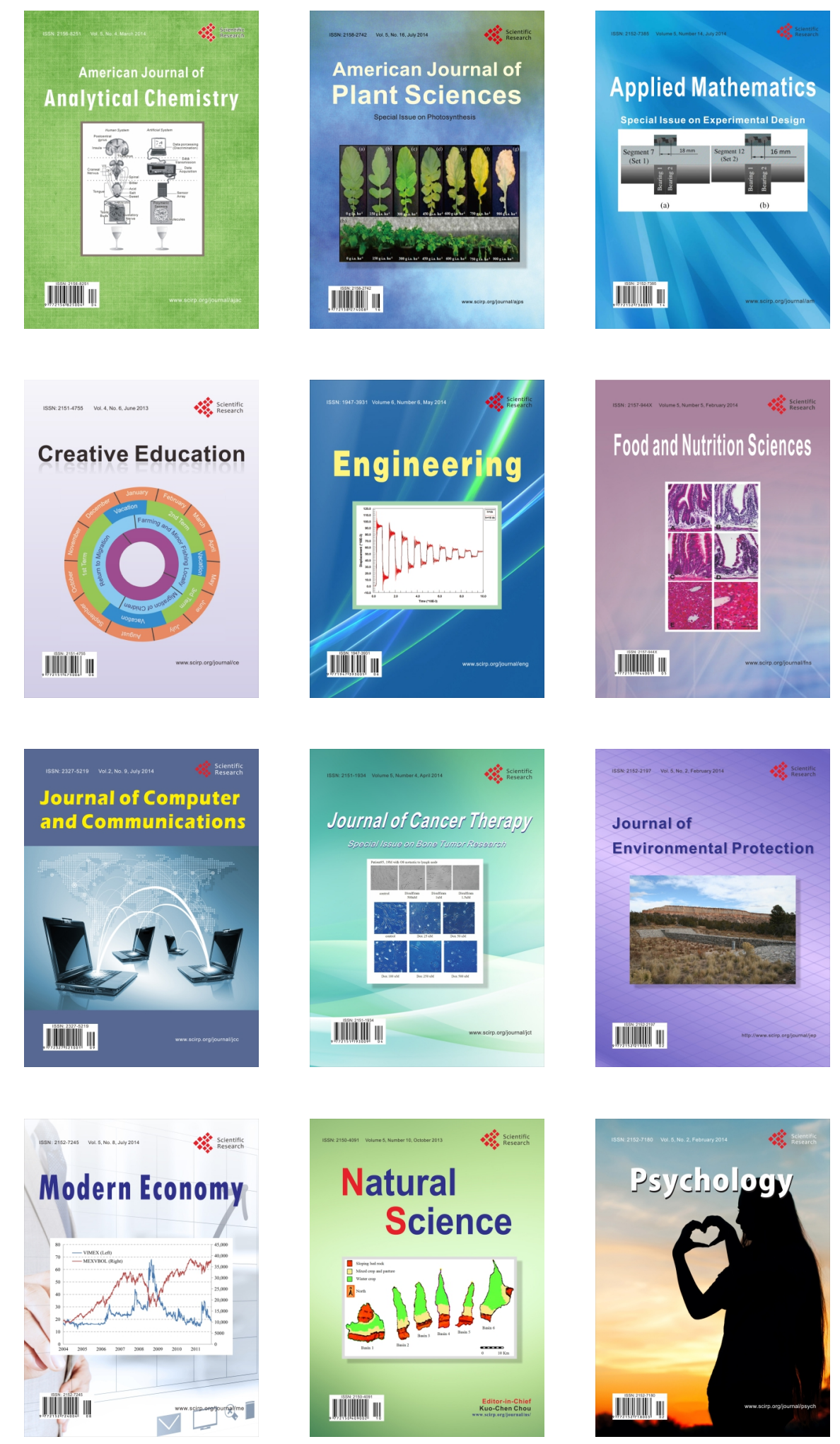